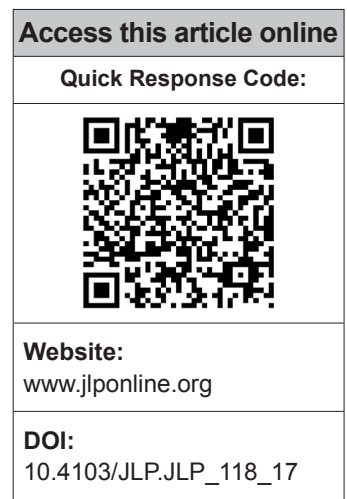

Department of Microbiology, NIMS,

${ }^{1}$ Department of

Microbiology, Kamineni Life Sciences, Hyderabad, Telangana, India

Address for correspondence: Dr. Neelima Angaali,

H.NO. 3-6-536,

Raja Residency,

Flat- 201, Street

No. 7, Himayat Nagar,

Hyderabad - 500 029,

Telangana, India.

E-mail: neelima sudharshan@gmail.com

Submission: 24-06-2017

Accepted: 13-11-2017

\title{
Direct identification and susceptibility testing of Gram-negative bacilli from turbid urine samples using VITEK2
}

\author{
Neelima Angaali, Laxmi Vemu', Chavali Padmasri, Neeraja Mamidi, Vijay Dharma Teja
}

\section{Abstract:}

INTRODUCTION: Urinary tract infections (UTIS) are the most common infectious diseases occurring in either the community or healthcare setting. Turnaround time for urine culture is about $24 \mathrm{~h}$, and antimicrobial susceptibility testing (AST) requires another $24 \mathrm{~h}$. Consequently, initial antibiotic therapy is mostly empirical.

MATERIALS AND METHODS: This study was conducted at Nizam's Institute of Medical Sciences, Hyderabad. Turbid urine samples which showed pus cells and Gram-negative (GN) bacilli of single morphotype were included. The turbidity of the urine was adjusted to $0.5 \mathrm{McF}$ arland and uploaded directly in the VITEK 2 identification (ID) GN and N-280 panel for AST. The specimen was also inoculated on CHROMagar, and the ID and AST of the isolates from the agar plate were repeated on VITEK 2, and the results were compared.

RESULTS: Out of 844 turbid urines screened, 62 met the inclusion criteria. Escherichia coli was the most common isolate $(71.9 \%)$. Complete agreement for ID was $80.7 \%$, misidentified were $12.2 \%$, and unidentified were $7 \%$. Complete agreement with AST was $94.3 \%$, very major errors $0.5 \%$, major errors $2.2 \%$, and minor errors $3 \%$.

CONCLUSION: With a $94.3 \%$ agreement for AST and a reduced turnaround time by $24 \mathrm{~h}$, the direct inoculation had a potential clinical benefit for initiating timely and appropriate antibiotic therapy for UTI.

Key words:

Complete agreement, major errors, minor errors, very major errors, VITEK 2 IDGN, VITEK 2 N280

\section{Introduction}

U rinary tract infections (UTIs) are among the most common infectious diseases occurring in either the community or healthcare setting. ${ }^{[1]}$ Urine culture needs up to $18-24 \mathrm{~h}$ of incubation, and if further testing is required, pathogen identification (ID) and antimicrobial susceptibility testing (AST) need an additional 24-48 h. ${ }^{[2]}$ Consequently, the initial antibiotic therapy is mostly empirical.

Based on the knowledge of the local spectrum of pathogens and the prevalence of antimicrobial resistance, UTIs are commonly treated empirically. ${ }^{[3]}$ Due to increasing

This is an open access journal, and articles are distributed under the terms of the Creative Commons Attribution-NonCommercial-ShareAlike 4.0 License, which allows others to remix, tweak, and build upon the work non-commercially, as long as appropriate credit is given and the new creations are licensed under the identical terms.

For reprints contact: reprints@medknow.com antibiotic resistance to commonly used oral agents, empiric treatment has no guaranteed effect on the pathogen. ${ }^{[3-5]}$

With inappropriate antibiotic prescriptions, healthcare cost increases and quality of care decreases. ${ }^{[6]}$ To avoid the use of broad-spectrum antibiotics and to optimize antibiotic treatment, microbiology laboratory should develop procedures with short turnaround time for AST. ${ }^{[5]}$

For this reason, microbiologists have for many years tried to reduce the turnaround time of bacterial ID and susceptibility testing. At the beginning, microscopic, chemical, and new automated methods provided rapid and cost-effective alternatives to standard culture

How to cite this article: Angaali N, Vemu L, Padmasri C, Mamidi N, Teja VD. Direct identification and susceptibility testing of Gram-negative bacilli from turbid urine samples using VITEK2. J Lab Physicians 2018;10:299-303. 
techniques for detecting bacteriuria. ${ }^{[2,7,8]}$ Gram staining of concentrated urine samples has demonstrated high sensitivity with high positive predictive value for the quick and cheap diagnosis of UTIs ${ }^{[9]}$ Recently, a real-time PCR method and matrix-assisted laser desorption/ionization time-of-flight mass-spectrometry (MALDI-TOF MS) have been described for the direct ID of bacterial species from urine samples. ${ }^{[10-12]}$ However, additional time is required for antibiotic susceptibility testing.

We studied a rapid method using the VITEK 2 system (Biomérieux), which has been widely introduced in most clinical microbiology laboratories, for ID and susceptibility testing of urinary tract pathogens directly from urine specimens of patients with suspected UTI selected by pyuria and screened by Gram staining and compared with the conventional method.

\section{Materials and Methods}

The study was conducted at Nizam's Institute of Medical Sciences from October 2016 to February 2017. A total of 4594 urine samples received to the microbiology laboratory were screened, out of which, 864 were turbid and 62 samples met the inclusion criteria.

\section{Inclusion criteria}

Turbid urine samples with turbidity $>0.5$ McFarland, Gram stain showing pus cells, Gram-negative (GN) bacilli of single morphotype.

\section{Exclusion criteria}

Absence of pus cells and organisms, $>$ one morphotype of GN Bacilli, candiduria, and turbidity $<0.5 \mathrm{McF}$ arland.

The turbid urine specimen fitting into the criteria was taken in a sterile test tube. Ten microliter was centrifuged in a sterile tube at $10,000 \mathrm{~g}$ for $10 \mathrm{~min}$. Supernatants were discarded. Ten microliter of the centrifuged urine specimen was spotted on a glass slide with a $0.01-\mathrm{mL}$ calibrated loop. Smears were air dried, heat fixed, Gram stained and examined under oil immersion for bacterial counts. The samples were directly inoculated onto VITEK 2 cards for ID and AST. The same sample were incoculated onto $\mathrm{CHROMagar}$ and incubated at $37^{\circ} \mathrm{C}$ for $24 \mathrm{~h}$. The next day, pure growth from the culture plate was inoculated onto VITEK 2 cards for ID and AST, and both the results were compared.

\section{Direct method}

The bacterial pellet was collected with a cotton swab to prepare a 0.5 McFarland suspension in $3.5 \mathrm{~mL}$ of $0.45 \%$ saline using the Densichek plus (Biomerieux).

At this point, the VITEK 2 cards were inoculated following the instructions of the manufacturer. Cards were selected depending on Gram stain analysis. When GN rods were observed, IDGN were used. AST-N280 (antimicrobial susceptibility testing) was the card employed for antibiotic susceptibility testing of GN bacilli.

\section{Standard inoculation method}

About $10-\mu \mathrm{L}$ volume of a urine sample was spread onto solid chromogenic agar media (CPS3; Biomérieux). The inoculated plates were incubated at $37^{\circ} \mathrm{C}$ for $24 \mathrm{~h}$. In cases of monomicrobial growth after incubation, a standardized 0.5 McFarland inoculum was prepared from the agar medium, and the VITEK 2 cards mentioned above were inoculated following the manufacturer's recommendations. The microbiological culture was considered as the reference method for ID and antibiotic susceptibility testing.

\section{Quality control}

Periodically, all cards studied were tested with ATCC Escherichia coli 25922 and ATCC Pseudomonas aeruginosa 27853.

\section{Data analysis}

Bacterial ID and susceptibility data from the direct method were compared with those obtained from the standard test.

The ID results with the direct method were grouped into three different categories:

1. Complete agreement: The strain ID with the direct VITEK 2 test and with the reference method was consistent at genus and species level

2. Misidentification: The strain ID with both systems was discrepant at the genus and the species level

3. Not identified: No ID was given at all.

Susceptibility results for all isolates tested were characterized into three clinical categories (susceptible [S], intermediate $[\mathrm{I}]$, or resistant $[\mathrm{R}]$ ) according to the criteria of the VITEK 2 advanced expert system.

Discrepancies were classified as follows:

1. Very major errors (VM): They were defined as an $S$ result with the direct VITEK 2 test and an $R$ result according to the reference method;

2. Major error (ME): They were defined as an $\mathrm{R}$ result with the direct VITEK 2 test and an $\mathrm{S}$ result according to the reference method.

3. Minor error (MinE): Any other category discrepancy was defined as a minor error (MinE).

Agreements were classified as follows:

1. Essential agreement (EA): Minimal Inhibitory Concentration (MIC) obtained for the direct method was within \pm 12 -fold dilution of the conventional $\operatorname{method}^{[13]}$ 
2. Categorical agreement (CA): MIC from direct method in the same susceptibility category as the conventional method $^{[13]}$

3. Precision EA (PEA): Agreement within $+/-12$-fold dilution of the precision test isolate antibiotic MIC

4. Precision CA (PCA): Agreement with the interpretative results of the precision test isolate using Clinical Laboratory Standard Institute (CLSI) criteria.

Based on the US Food and Drug Administration (FDA)/CLSI approved criteria, the overall ME $\leq 5 \%$ is acceptable, EA and CA $\geq 90 \%$, PEA and PCA $\geq 95 \%$, $\mathrm{ME}$ and $\operatorname{MinE}<10 \%{ }^{[13]}$

To assess the precision of ID and susceptibility five test samples with three ATCC strains of E. coli, Klebsiella pneumoniae, and $P$. aeruginosa were run in triplicate daily for 5 days.

The following antimicrobial agents were compared: Amoxicillin-clavulanic acid, ampicillin, piperacillin-tazobactam, cefuroxime, cefuroxime axetil, ceftriaxone, cefoperazone-sulbactam, cefepime, ciprofloxacin, ertapenem, imipenem, meropenem, gentamicin, amikacin, nalidixic acid, nitrofurantoin, and trimethoprim-sulfamethoxazole [Table 1].

\section{Results}

A total of 844 turbid urines received to microbiology laboratory were screened, out of which, 62 samples met the inclusion criteria. All the samples which met the inclusion criteria were processed by both routine and direct methods. Among these 62 samples, polymicrobial growth was observed in 3 samples,

Table 1: Concentration ranges of the used antimicrobial agents (antimicrobial susceptibility testing N280)

\begin{tabular}{lc}
\hline Antimicrobial agent & Concentration range $(\mu \mathrm{g} / \mathrm{ml})$ \\
\hline Amikacin & $2-64$ \\
Amoxicillin/clavulanic acid & $2 / 1-32 / 16$ \\
Ampicillin & $2-32$ \\
Cefepime & $1-645$ \\
Cefoperazone/sulbactam & $8-64$ \\
Ceftriaxone & $1-64$ \\
Cefuroxime & $1-64$ \\
Ciprofloxacin & $0.25-4$ \\
Colistin & $0.5-16$ \\
Ertapenem & $0.5-8$ \\
Gentamicin & $1-16$ \\
Imipenem & $0.25-16$ \\
Meropenem & $0.25-16$ \\
Nalidixic acid & $2-32$ \\
Nitrofurantoin & $16-512$ \\
Piperacillin/tazobactam & $4 / 4-128 / 4$ \\
Trimethoprim/sulfamethoxazole & $20-320$ \\
\hline
\end{tabular}

and two samples were sterile, so these 5 samples were excluded, and finally, 57 samples were analyzed. E. coli was the predominant isolate $(41,71.92 \%)$, followed by K. pneumoniae $(10,17.54 \%)$. These microorganisms of the Enterobacteriaceae family could be accurately identified directly from urine within $4.5 \mathrm{~h}$.

The overall complete agreement for ID was $80.7 \%$ [Table 2]. Of these 57 isolates, 7 were misidentified (12.2\%) and 4 were unidentified $(7 \%)$ by direct method. Out of the 7 misidentified isolates, 5 were E. coli and 2 were K. pneumoniae. E. coli was misidentified as Rauotella, Sphingomonas, Yersinia, and Serratia, and K. pneumoniae was misidentified as K. oxytoca.

The isolates which showed complete agreement for ID were analyzed for AST agreement. About 46 isolates showed complete agreement and these isolates were evaluated for susceptibility EA, complete agreement (Categorical agreement (CA); sensitive, intermediate, resistant), VM error, major error (ME), minor error (MinE), and precision.

For the 46 g negative isolates AST, EA was $97.3 \%$ and CA $94.3 \%$. EA was $100 \%$ for the 7 antimicrobial drugs, $97.8 \%$ for 4 drugs. CA was $100 \%$ for the 3 antimicrobial drugs [Table 3].

VM errors were reported with cefepime, ertapenem, and imipenem. All were from the same isolate K. pneumoniae. Major errors were commonly reported for piperacillin-tazobactam followed by ertapenem, imipenem, meropenem, and cotrimoxazole. These major errors were reported in E. coli, K. pneumoniae, and $P$. aeruginosa. Minor errors were reported commonly for piperacillin-tazobactam and nitrofurantoin followed by amoxyclav. Combined error rate was 5.62\%.

PEA and PCA were $99 \%$ for all drugs combined.

\section{Discussion}

This study was done to assess the reliability of the VITEK 2 system for direct ID and antibiotic susceptibility testing of urinary tract pathogens (directly) from the urine sediment. E. coli was the predominant isolate $(71.92 \%)$ which was comparable (56.8\%) to the study conducted by Niranjan and Malini. ${ }^{[14]}$

The results of this study showed that the microorganisms of the Enterobacteriaceae family, most often responsible for the urinary infection, can be accurately identified directly from urine within $4.5 \mathrm{~h}$ using the VITEK 2 direct system, with $80.7 \%$ correlation with the standard technique. Compared with other ID systems directly from urine specimens, the $80.7 \%$ correlation rate for 
Enterobacteriaceae is similar or slightly lower than that obtained by others. ${ }^{[10,11,15,16]}$

Overall, 7 microorganisms were misidentified or not identified by the direct method while it was 19 in the study conducted by Munoz-Dávila et al. ${ }^{[17]}$

Cellular elements, inhibitory substances, or $\mathrm{pH}$ changes in urine could have contributed to the false-positive and false-negative reactions responsible for misidentification.

In addition, the criteria stated by Jorgensen and Ferraro and those from the CLSI and FDA recommend that a new test method should provide $90 \%$ EA with MIC values determined by the reference method. ${ }^{[18]}$ The susceptibility CA was $94.3 \%$ and EA was $97.3 \%$ rate found in this study which was similar to Munoz-Dávila et al. ${ }^{[17]}$

VM errors, major error, and minor errors of $0.5 \%$, $2.2 \%, 3 \%$, respectively, were reported in this study. Munoz-Dávila et al. reported 0.2\% VM errors and $0.4 \%$ major errors. ${ }^{[17]}$

Table 2: Identification of Gram-negative isolates by routine and direct method

\begin{tabular}{lccc}
\hline Name of the isolate & Conventional & Direct & Percentage \\
\hline Escherichia coli & 41 & 32 & 78.04 \\
Klebsiella pneumoniae & 10 & 8 & 80 \\
Klebsiella oxytoca & 1 & 1 & 100 \\
Pseudomonas aeruginosa & 2 & 2 & 100 \\
Morganella morganii & 1 & 1 & 100 \\
Enterobacter cloacae & 1 & 1 & 100 \\
Acinetobacter & 1 & 1 & 100 \\
Total & 57 & 46 & 80.7 \\
\hline
\end{tabular}

The combined ME and MinE rate was $5.6 \%$ while it was $1.04 \%$ in the study conducted by Bazzi et al. ${ }^{[19]}$

In summary, the results of this study indicate that the organisms can be accurately identified directly from an inoculum prepared from centrifuged urine specimens. Susceptibility test data may also be obtained that compare favorably with routine VITEK 2 susceptibility results performed on isolated colonies of the same organism. This method permits the reporting of the complete results on urine specimens with GN bacilli as early as $8 \mathrm{~h}$ after their arrival in the laboratory. The most important benefit is the faster pathogen ID and susceptibility data, which allow earlier selective antimicrobial therapy. The impact of rapid procedures on patient care, laboratory efficiency, and cost containment is significant. ${ }^{[6]}$

This direct method could be very useful in those microbiology laboratories with a high number of urine specimens from the emergency department (highly), which employ the VITEK 2 system as the reference method for bacterial ID and antibiotic susceptibility testing. This direct test might be an excellent alternative to the automated urine screening systems and to the MALDI-TOF MS technology, both techniques require special hardware therefore high investment costs for most laboratories. ${ }^{[20]}$

However, before inoculating the VITEK 2 card, several data had to be collected (microscopy of the primary urine, selecting for monomorphology, and bacterial count).

\section{Limitations of the study}

The study was conducted with a smaller sample size, because of this low number, further studies are necessary

Table 3: Antimicrobial susceptibility testing agreement between direct and standard for Gram-negative bacteria using VITEK N280

\begin{tabular}{|c|c|c|c|c|c|c|}
\hline Antibiotic & Very major & Major error & Minor error & CA & EA & Total \\
\hline Ampicillin & 0 & 0 & 1 & 45 & 46 & 46 \\
\hline Amoxyclav & 0 & 0 & 3 & 43 & 46 & 46 \\
\hline Piperacillin-tazobactam & 0 & 3 & 5 & 38 & 43 & 46 \\
\hline Cefuroxime & 0 & 1 & 2 & 43 & 45 & 46 \\
\hline Ceftriaxone & 0 & 0 & 3 & 43 & 46 & 46 \\
\hline Cefoperazone-sulbactam & 0 & 1 & 2 & 43 & 45 & 46 \\
\hline Cefepime & 2 & 1 & 2 & 41 & 43 & 46 \\
\hline Ertapenem & 1 & 2 & 0 & 43 & 43 & 46 \\
\hline Imipenem & 1 & 2 & 0 & 43 & 43 & 46 \\
\hline Meropenem & 0 & 2 & 1 & 43 & 43 & 46 \\
\hline Amikacin & 0 & 1 & 0 & 45 & 45 & 46 \\
\hline Gentamicin & 0 & 1 & 0 & 45 & 45 & 46 \\
\hline Nalidixic acid & 0 & 0 & 0 & 46 & 46 & 46 \\
\hline Ciprofloxacin & 0 & 0 & 0 & 46 & 46 & 46 \\
\hline Nitrofurantoin & 0 & 0 & 5 & 41 & 46 & 46 \\
\hline Colistin & 0 & 0 & 0 & 46 & 46 & 46 \\
\hline Cotrimoxazol & 0 & 2 & 0 & 44 & 44 & 46 \\
\hline Total (\%) & $4(0.5)$ & $16(2.2)$ & $24(3)$ & 738 (94.3) & 761 (97.3) & 782 (100) \\
\hline
\end{tabular}


to assess the reliability of the VITEK 2 system for direct ID of other GN, nonfermentative rods and Gram-positive cocci.

\section{Conclusion}

With a $94.3 \%$ agreement for AST for common urinary tract pathogens and a reduced turnaround time by $24 \mathrm{~h}$, the direct inoculation of the VITEK 2 system had a potential clinical benefit for initiating timely and appropriate antibiotic therapy for UTI. This could benefit both patient and hospital in providing better treatment and reducing the cost.

\section{Financial support and sponsorship Nil.}

\section{Conflicts of interest}

There are no conflicts of interest.

\section{References}

1. Peleg AY, Hooper DC. Hospital-acquired infections due to gram-negative bacteria. N Engl J Med 2010;362:1804-13.

2. Graham JC, Galloway A. ACP best practice no 167: The laboratory diagnosis of urinary tract infection. J Clin Pathol 2001;54:911-9.

3. Hoban DJ, Lascols C, Nicolle LE, Badal R, Bouchillon S, Hackel M, et al. Antimicrobial susceptibility of Enterobacteriaceae, including molecular characterization of extended-spectrum beta-lactamase-producing species, in urinary tract isolates from hospitalized patients in North America and Europe: Results from the SMART study 2009-2010. Diagn Microbiol Infect Dis 2012;74:62-7.

4. Breteler KB, Rentenaar RJ, Verkaart G, Sturm PD. Performance and clinical significance of direct antimicrobial susceptibility testing on urine from hospitalized patients. Scand J Infect Dis 2011;43:771-6.

5. Kotwani A, Holloway K. Trends in antibiotic use among outpatients in New Delhi, India. BMC Infect Dis 2011;11:99.

6. Galar A, Yuste JR, Espinosa M, Guillén-Grima F, Hernáez-CrespoS, Leiva J, et al. Clinical and economic impact of rapid reporting of bacterial identification and antimicrobial susceptibility results of the most frequently processed specimen types. Eur J Clin Microbiol Infect Dis 2012;31:2445-52.

7. Washington JA, White IC, Laganiere M, Smith L. Detection of significant bacteriuria by microscopic examination of urine. Lab
Med 1981;12:294-6.

8. Lamb VA, Dalton HP, Wilkins JR. Electrochemical method for the early detection of urinary-tract infections. Am J Clin Pathol 1976;66:91-5.

9. Cardona N, Rojas C, Zabalaga L. Leukocytes in urine and gram tint for the diagnosis of urinary infection. Rev Soc Boliviana Pediatr 2008;47:81-5.

10. Lehmann LE, Hauser S, Malinka T, Klaschik S, Weber SU, Schewe JC, et al. Rapid qualitative urinary tract infection pathogen identification by SeptiFast real-time PCR. PLoS One 2011;6:e17146.

11. Ferreira L, Sanchez-Juanes F, Gonzalez-Avila M, Cembrero-Fucinos D, Herrero-Hernandez A, Gonzalez-BuitragoJM, et al. Direct identification of urinary tract pathogens from urine samples by matrix-assisted laser desorption ionization-time of flight mass spectrometry. J Clin Microbiol 2010;48:2110-5.

12. Köhling HL, Bittner A, Müller KD, BuerJ, Becker M, Rübben H, et al. Direct identification of bacteria in urine samples by matrix-assisted laserdesorption/ionization. Time-of-flight mass spectrometry and relevance of defensins as interfering factors. J Med Microbiol 2012;61:339-44.

13. Clark RB, Lewinski MA, Loeffelholz MJ, Tibbetts RJ. Cumitech31a: Verification and validation of procedures in the clinical microbiology laboratory. In: Sharp SE, editor. Coordinating. Washington, DC: ASM Press; 2009.

14. Niranjan V, Malini A. Antimicrobial resistance pattern in Escherichia coli causing urinary tract infection among inpatients. Indian J Med Res 2014;139:945-8.

15. Ilki A, Bekdemir P, Ulger N, Soyletir G. Rapid reporting of urine culture results: Impact of the uro-quick screening system. New Microbiol 2010;33:147-53.

16. Marschal M, Wienke M, Hoering S, Autenrieth IB, Frick JS. Evaluation of 3 different rapid automated systems for diagnosis of urinary tract infections. Diagn Microbiol Infect Dis 2012;72:125-30.

17. Munoz-Dávila MJ, Roig M, Yagüe G, Blázquez A, Salvador C, Segovia M. Comparative evaluation of Vitek 2 identification and susceptibility testing of urinary tract pathogens directly and isolated from chromogenic media. Eur J Clin Microbiol Infect Dis 2013;32:773-80.

18. Jorgensen JH, Ferraro MJ. Antimicrobial susceptibility testing: General principles and contemporary practices. Clin Infect Dis 1998;26:973-80.

19. Bazzi AM, Rabaan AA, Fawarah MM, Al-Tawfiq JA. Direct identification and susceptibility testing of positive blood cultures using high speed cold centrifugation and Vitek II system. J Infect Public Health 2017;10:299-307.

20. Ferreira L, Vega S, Sánchez-Juanes F, González M, Herrero A, Muñiz MC, et al. Identifying bacteria using a matrix-assisted laser desorption ionization time-of-flight (MALDI-TOF) mass spectrometer. Comparison with routine methods used in clinical microbiology laboratories. Enferm Infecc Microbiol Clin 2010;28:492-7. 